\title{
Coevolving nonlinear voter model with triadic closure
}

\author{
Tomasz Raducha ${ }^{1,2}$ (a), Byungjoon Min ${ }^{2,3}$ and Maxi San Miguel ${ }^{2}$ (b) \\ 1 Institute of Experimental Physics, Faculty of Physics, University of Warsaw, Pasteura 5, 02-093 Warsaw, Poland \\ 2 IFISC, Instituto de Física Interdisciplinar y Sistemas Complejos (CSIC-UIB), Campus Universitat Illes Balears, \\ E-07122 Palma de Mallorca, Spain \\ 3 Department of Physics, Chungbuk National University, Cheongju, Chungbuk, 28644 South Korea
}

PACS $05.70 . \mathrm{Ln}$ - Nonequilibrium and irreversible thermodynamics
PACS $89.65 .-\mathrm{s}$ - Social and economic systems
PACS $89.75 . \mathrm{Hc}$ - Networks and genealogical trees

Abstract -We study a nonlinear coevolving voter model with triadic closure local rewiring. We find three phases with different topological properties and configuration in the steady state: absorbing consensus phase with a single component, absorbing fragmented phase with two components in opposite consensus states, and a dynamically active shattered phase with many isolated nodes. This shattered phase, which does not exist for a coevolving model with global rewiring, has a lifetime that scale exponentially with system size. We characterize the transitions between these phases in terms of the size of the largest cluster, the number of clusters, and the magnetization. Our analysis provides a possible solution to reproduce isolated parts in adaptive networks and high clustering widely observed in social systems.

Introduction. - Network dynamics [1, 2, 3, 4, 5, often refers to the dynamics of the network creation or evolution, but also to the dynamics on the network, where the dynamics of the states of the nodes is affected by the topological structure of a fixed underlying network. However, the structure of the network among interacting agents evolves dynamically in response to the state of the nodes. Therefore, dynamics of and on the network are dynamically coupled processes occurring in comparable time scales. The coupling of these processes has been referred as Coevolution of node states and network structure [6, 7].

In order to understand this coevolutionary dynamics of complex systems, there have been several studies which incorporate both the change of networks and the dynamical processes on networks [8, 9, 10, 11, 12]. Coevolutionary dynamics has been applied in a variety of different fields ranging form classical spin models [12, 13, opinion formation [8, 10, 14, game theory 7 and epidemic spreading [9, 15, 16, 17], to cultural dynamics [18, 19, 20, 21, and ecological interactions 22,23 .

A prototype coevolving model is the Coevolution Voter Model (CVM) combining the voter model and the change of a network by rewiring links [8, 10]. In the original voter

\footnotetext{
(a) tomasz.raducha@fuw.edu.pl

(b) maxi@ifisc.uib-csic.es
}

model [24, 25, 26, a node can be in one of two states, called up and down. At each step, each node adopts the state of one of its neighbors chosen randomly. Adding link rewiring to the original voter model, a coevolving voter model was addressed [8, 10]. In a coevolving voter model, in addition to node's dynamics following the voter model, the links in a network can be updated. In a typical CVM, a global rewiring of links is adopted for the sake of simplicity, meaning that a newly connected node is chosen randomly out of all nodes in a whole network. The coevolving voter model shows a generic absorbing phase transition between a connected and fragmented network 8. However, the global rewiring might be unrealistic since there should be some limitation of the range of searching for a node to be connected. Many observations indicate that a link rewiring in networks is mainly implemented by the process of triadic closure, i.e. the tendency that nodes search for new contacts through existing neighbors [27, 28, 29]. In this regard, the effect of triadic closure was analyzed in many models of social dynamics [29, 30, 31, 32]. CVM with rewiring by triadic closure was also studied 33 to describe the community structure of an online society. Triadic closure gives rise to a shattering phase 34 where many lonely nodes separate from a large connected component.

Recently, in addition to triadic closure another variant 
of CVM was proposed, incorporating collective behaviors between connected nodes in terms of nonlinearity of interactions [35]. The nonlinearity means that individuals can take into account the state of all of their neighbors as a whole, instead of a dyadic interaction, so that their action does not have to be proportional to the aggregated state of their neighbors. Modeling of this type of behavior goes generally under the name of Nonlinear Voter Model [36, 37, 38, 39, 40, 41, 42. In case of nonlinearity, diverse phases depending on the nonlinearity were found with the different mechanisms of network fragmentation [35]. A similar form of nonlinearity was considered in social impact theory [4], in language competition dynamics, called volatility [44, 45], or in language evolution problems [46. However, the effect of nonlinearity on coevolutionary dynamics has been explored only for a global link rewiring as the simplest example.

In this work, we study a coevolving nonlinear voter model 35] with a local rewiring making triadic closure [33. These two factors, nonlinearity and triadic closure, had been previously analyzed changing the output of the standard coevolving voter model. But, the two effects have not been,so far, taken into account together. We focus on a coevolving nonlinear voter model with a local rewiring where individuals interact with their neighbors in a nonlinear manner and a new neighbor can be chosen from among nodes distant by two edges, i.e. neighbors of neighbors. We numerically find three possible phases with different topological properties and magnetization: absorbing consensus phase with a single component, absorbing fragmented phase with two components of opposite states, and a dynamically active shattered phase with a coexistence of both states within the main component and a significant part of isolated nodes.

Model. - In our coevolving nonlinear voter model with triadic closure, coevolution is characterized by a plasticity parameter $p$ defining the ratio between the timescale of changes in the network topology and the timescale of the dynamics of nodes' states. A nonlinearity parameter $q$ measures the nonlinear effect of local majorities in the imitation mechanism of the voter model. Local rewiring means that when a link from an active node is suppressed, a new link from this node can only be created with neighbors of its neighbors, without creating multiple- or autoconnections.

Our detailed algorithm is as follows. We start every simulation with Erdös-Rényi (ER) graphs [47] with a mean degree $\langle k\rangle=8$. Every node is initially in one of two possible states $s_{i}= \pm 1$ with an equal probability $1 / 2$. The number of active links $a_{i}$ of a node $i$ is defined as the number of connections to nodes in a different state $s_{j} \neq s_{i}$. Consequently, we define the density of active links $\rho_{i}$ as the fraction of active links $\frac{a_{i}}{k_{i}}$, where $k_{i}$ is the degree of node $i$. In every time step a node $i$ is randomly selected from the network (Fig. 11. With probability $\rho_{i}^{q}$, where $q$ is a nonlinearity parameter, the focal node $i$ interacts with
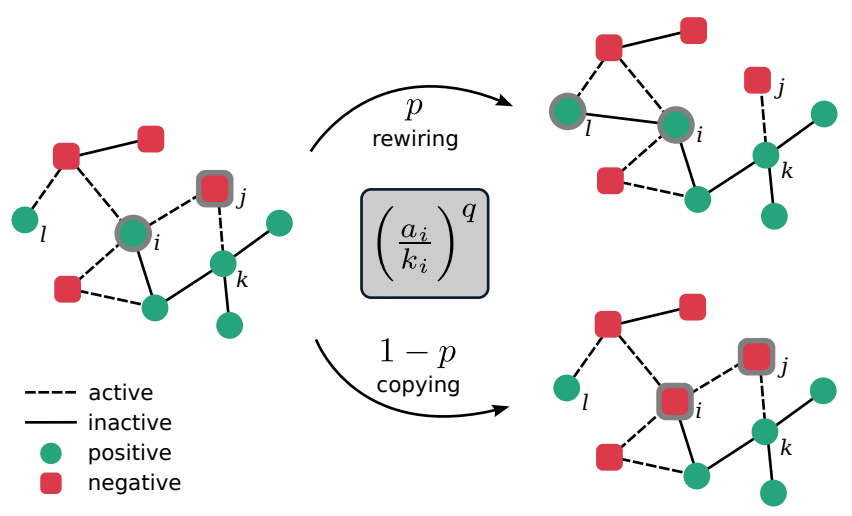

Fig. 1: Schematic illustration of update rules in a nonlinear coevolving voter model with triadic closure. Every node is in a state +1 (green) or -1 (red). The focal node $i$ is chosen randomly. Then with probability $\left(\frac{a_{i}}{k_{i}}\right)^{q}$ one of the active links to the node $j$ is chosen. With probability $p$ the link $(i-j)$ is rewired to link $(i-l)$ (as in the figure) or to link $(i-k)$. With probability $1-p$ the focal node $i$ copies the state of node $j$.

its neighbors. If node $i$ interacts, one of the active links to a neighbor $j$ is chosen at random. Next, with probability $p$ the active link is rewired, and with probability $1-p$ the focal node changes its state to the same state of node $j$. In the rewiring process the chosen active link is suppressed and a new one is created linking $i$ to a node distant by two links (but not less) and being in the same state as node $i$. If no such node exists, nothing happens. Obviously, the number of nodes $N$ and number of links $M$ is constant in time. This triadic closure process is repeated until a dynamically active stationary state is obtained or an absorbing configuration is reached.

If the plasticity parameter $p=0$ there is no rewiring. Therefore, the network configuration stays constant throughout the simulation, and fragmentation or shattering are not possible. On the other hand, for $p=1$ nodes do not change states so that consensus cannot be obtained, and links are rewired until the network undergoes a fragmentation transition into two separate components with opposite states (with possible detached nodes). The nonlinearity parameter $q$ defines the likelihood of interacting with individuals in the opposite state. For $q=1$ the model becomes the original coevolving voter model of random imitation of a neighbor. When $q>1$, nodes with more active links have a higher chance to change their state than in the ordinary voter model where this frequency is proportional to the number of active links. In contrast, if $q<1$, nodes with less active links are more likely to change their state than in the ordinary voter model.

Phase diagram: consensus, fragmentation, and shattering phases. - Our simulations are described in terms of three quantities: the magnetization $m=\frac{1}{N} \sum_{i} s_{i}$, 

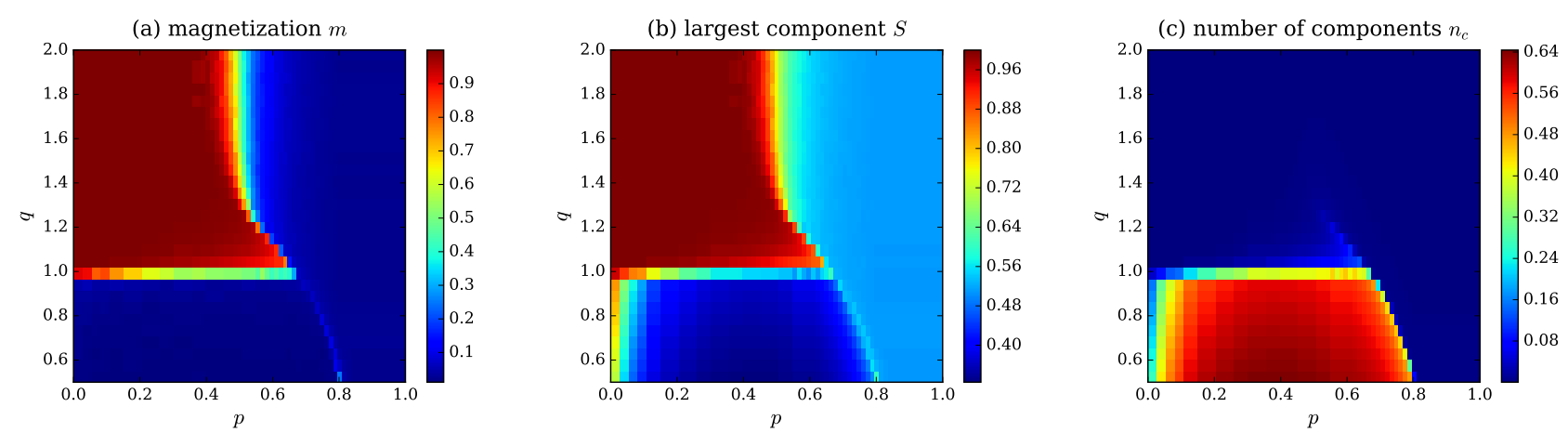

Fig. 2: Phase diagram in $(p, q)$ space. (a) Magnetization, (b) the size of the largest component, and (c) the number of components on ER networks with $N=5000, M=20000$, averaged over 500 simulation runs.

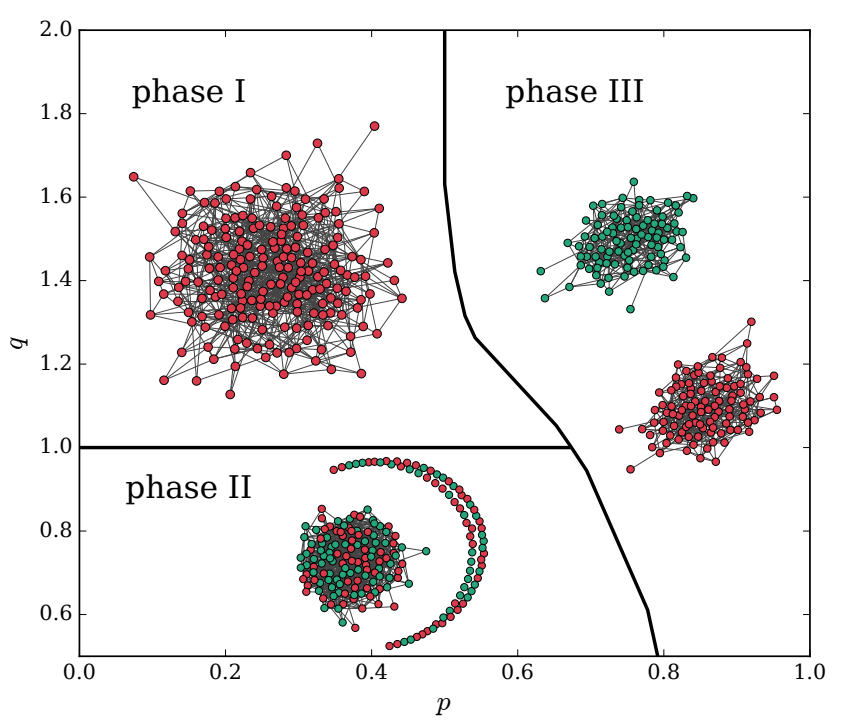

Fig. 3: Scheme of the phase diagram with respect to $p$ and $q$. Different phases are separated by a black line and for each a typical final configuration is presented, with opposite states colored in red and green.

the relative size of the largest connected component $S$ of the network, and the relative number of separate components $n_{c}$. Phase diagrams for these quantities in the $(p, q)$ parameter space are shown in Fig. 2. We find three different steady-state phases. For $q>1$ and $p<p_{c}(q)$, where $p_{c}(q)$ is the transition line separating the phase III from others, phase I - an absorbing consensus phase - is characterized by a non-zero value of the absolute magnetization and a single large component, $\left(|m|, S, n_{c}\right) \approx(1,1,0)$. When $q<1$ and $p<p_{c}(q)$, we obtain phase II, a shattered phase, where the absolute magnetization drops to zero and the network is composed of an active component and a number of shattered nodes. In the shattered phase, many nodes that initially belong to a connected component become shattered into isolated nodes, resulting in a high value of $n_{c}$. Therefore, the phase II can be identified as $\left(|m|, S, n_{c}\right) \approx\left(0, S^{*}, n_{c}^{*}\right)$ where $S^{*}$ is the size of a large active component of the network and $n_{c}^{*}$ is the number of shattered nodes. In our parameter sets, $S^{*}$ and $n_{c}^{*}$ are respectively approximately 0.4 and 0.6 . Increasing the value of $p$ above $p_{c}\left(p>p_{c}\right)$ for any $q$, we obtain phase III, a fragmented phase characterized by $\left(|m|, S, n_{c}\right) \approx(0,1 / 2,0)$. In phase III, the network fragments in two components of approximately the same size and each component in an opposite consensus state, so that the absolute magnetization is close to zero and the size of the largest component is around $1 / 2$.

The topological properties of the three different phases are illustrated in an example of a network configuration in the steady state (Fig. 3). First, in the consensus phase (phase I), a network is formed by a single connected component within a full consensus either $m=1$ or $m=-1$ in the steady state. Second, in the shattered phase (phase II), we find even more than a half of the nodes being isolated. In addition, the main cluster remains active, mixing up and down states of nodes at the steady state. This shattered phase is different from the active state with a connected component observed in the nonlinear voter model with global rewiring with similar parameters $(p, q)$ [35]. Last, in the fragmented phase (phase III) the network is polarized: two internally coherent clusters in opposite states.

\section{Fragmentation and shattering phase transitions.} - In the coevolving nonlinear model with triadic closure, phase transitions between different phases can be identified by examining $\left(|m|, S, n_{c}\right)$ as a function of $p$ and $q$ (Fig. 4). When $q<1$ with varying $p$, we find a phase transitions between the shattering (phase I) and fragmentation (phase III) phases [Fig. 4(a)]. At the critical point $p_{c}$, a shattered network with many detached nodes starts to recombine into two clusters with increasing $p$. In other words, a network with many isolated nodes and an active component transforms into two connected components with polarized configuration. The transition can be identified by the sharp drop of $n_{c}$ with increasing $p$. Note again that the shattered phase is different from the coexis- 
(a)

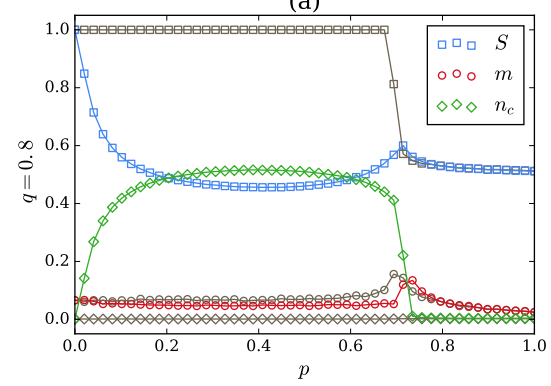

(d)

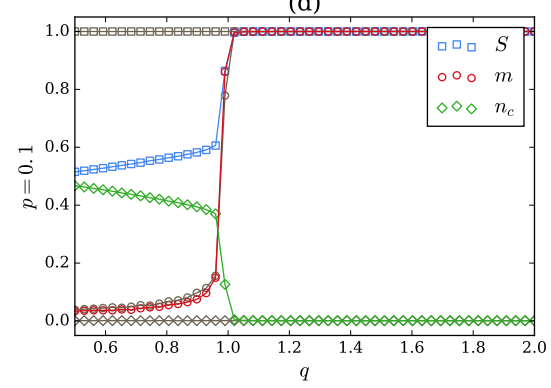

(b)

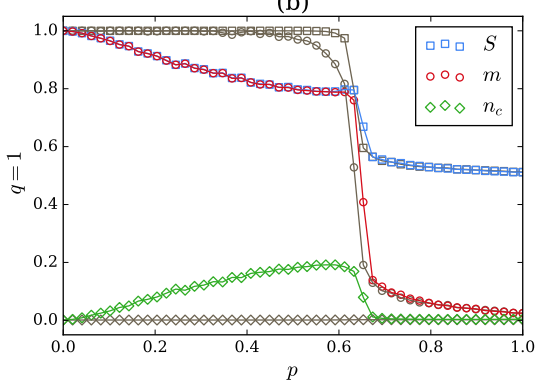

(e)

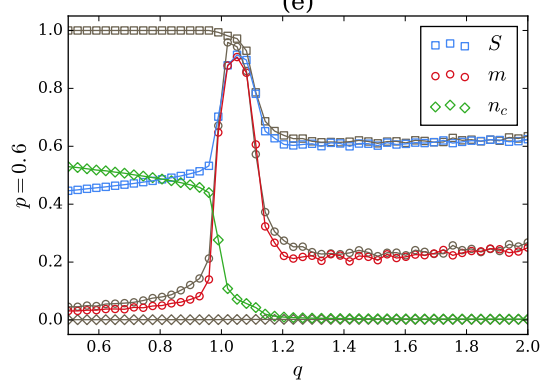

(c)

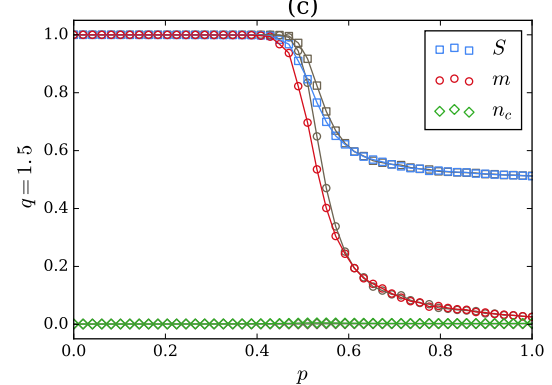

(f)

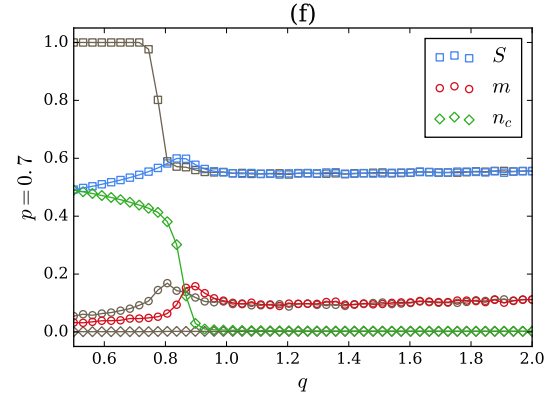

Fig. 4: Phase transition with respect to $p$ and fixed values of $q$ (a) $q=0.8$, (b) $q=1$, and (c) $q=1.5$, and with respect to $q$ and fixed values of $p$ (d) $p=0.1$, (e) $p=0.6$, and (f) $p=0.7$. The size of the largest component $S$ (blue squares), the magnetization $m$ (red circles), and the number of components $n_{c}$ (green diamonds) on ER networks with $N=1000, M=4000$, averaged over 500 simulation runs. Every quantity for global rewiring [35] is also plotted with the same symbols in gray.

tence phase observed in an ordinary coevolving nonlinear voter model with global rewiring the network [35] when $q<1$ and $p<p_{c}$, as shown in the differences in $S$ and $n_{c}$.

When $q>1$, we find a fragmentation transition between the consensus (phase I) and fragmented (phase III) phases, also observed in the ordinary CVM and nonlinear CVM [Fig. 4(c)]. Increasing $p$, the network splits from a single component with a full consensus state into two separate clusters in opposite consensus states. For $p>p_{c}$, the magnetization $|m|$ and the number of components $n_{c}$ approach to zero, and the size of the largest component $S$ tends to 0.5 . The fragmented phase (phase III) can be obtained for any value of $q$ if the plasticity parameter $p$ is higher than $p_{c}(q)$. Thus, when $p>p_{c}$, the behaviors of $S$, $|m|$, and $n_{c}$ for different $q$ share common features.

In Fig. 4(d-f), we show $S,|m|$, and $n_{c}$ as a function of $q$ for fixed values of $p(p=0.1,0.6,0.7)$. For $p=0.1$ as a representative example of $p<p_{c}$ [Fig. 4(d)], by varying $q$ we observe an absorbing phase transition between phase I and phase II. For $q \geq 1$, numerical simulations always reach a dynamically frozen state. For $q<1$, however we find a dynamically active phase with a high fraction of isolated nodes and an active cluster showing coexistence of up and down states of nodes. This shattered phase observed for local rewiring is a new phenomenon that was not detected for global rewiring.

For $p=0.6$ [Fig. 4(e)], varying $q$ we can observe all three phases and two transitions. First, for small $q$ we observe the active shattered phase. Increasing $q$ above 1 we first obtain a transition to the consensus phase with high magnetization $|m|$ and a high value of the largest component size $S$. The second fragmentation transition occurs for larger values of $q$ to the fragmented phase with low values of $|m|$ and $S \approx 1 / 2$. When $p$ increases further, i.e, $p=0.7$ [Fig. 4(f)], the consensus phase disappears and the shattered phase changes directly to the fragmented phase as $q$ increases, as indicated by a decreasing value of $n_{c}$ at the transition.

Convergence time. - A finite system is always bound to reach an absorbing phase for any combination of parameters in the limit $t \rightarrow \infty$. But for the phase II, which is an active phase, the convergence time $\tau$ to an absorbing state grows exponentially with increasing system size $N, \tau \sim e^{N}$ as in the case of global rewiring [35]. Exponential divergence of $\tau$ in Monte-Carlo steps for phase II is clearly shown in Fig. 5(a). Therefore, it is expected to remain the active phase rather than to reach an absorbing state in a finite time in the limit $N \rightarrow \infty$ when $q<1$ and $p<p_{c}$. For $q=1$ (corresponding to a linear interaction) and $p<p_{c}$, we reproduce a linear scaling with system size $N, \tau \sim N$, see Fig. 5(b), as reported also in the linear CVM and nonlinear CVM with global rewiring [8, 35]. We also find that the convergence to the frozen state with $q=1.5$ slows down at the critical point $p_{c}[\mathrm{Fig} .5(\mathrm{c})]$. 

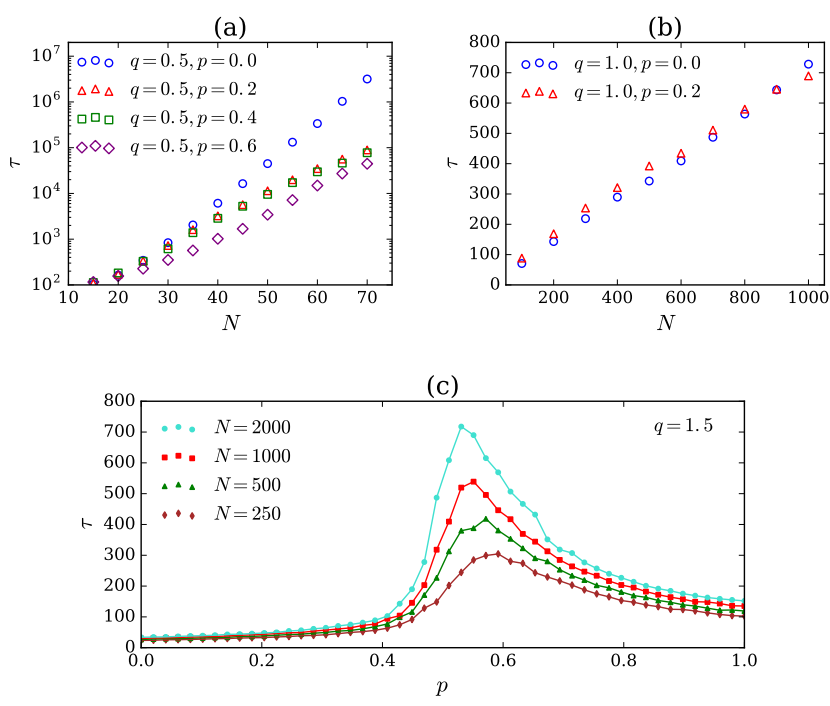

Fig. 5: Convergence time $\tau$ in the number of Monte Carlo steps to a frozen configuration as a function of network size $N$ in (a) the phase II and at (b) $q=1$. (c) Convergence time $\tau$ as a function of $p$ for $q=1.5$ (phases I and III). Results are obtained for ER networks with $\langle k\rangle=8$, averaged over 500 realizations.

Clustering coefficient. - We finally investigated the internal structure of networks generated in the model focusing on the average clustering coefficient $\langle C\rangle$. For a node $i$ with degree $k_{i}$, the local clustering coefficient $C_{i}$ is defined as 48

$$
C_{i}=\frac{2 L_{i}}{k_{i}\left(k_{i}-1\right)}
$$

where $L_{i}$ is the number of links between the $k_{i}$ neighbors of node $i$. Then, the average clustering coefficeint representing the average of $C_{i}$ over all nodes is given by

$$
\langle C\rangle=\frac{1}{N} \sum_{i=1}^{N} C_{i}
$$

In Fig. 6 we present average local clustering coefficient for different values of $(p, q)$ in the steady state. For nodes having less than two links, local clustering coefficient is not well defined, and for that reason we exclude these nodes in our analysis. While the value of the clustering coefficient for global rewiring remains almost zero for all tested parameter sets, it clearly shows values far from zero, exceeding 0.3 in an extreme case, for local rewiring. In addition, the location of peaks in $\langle C\rangle$ is coincident with the transition point, meaning that the clustering coefficient can be an indicator of phase transitions. Considering the fact that real-world social systems are known to display high clustering [4, 27, 29, our model can provide a plausible way to reconstruct such structure aiming to describe social phenomena.
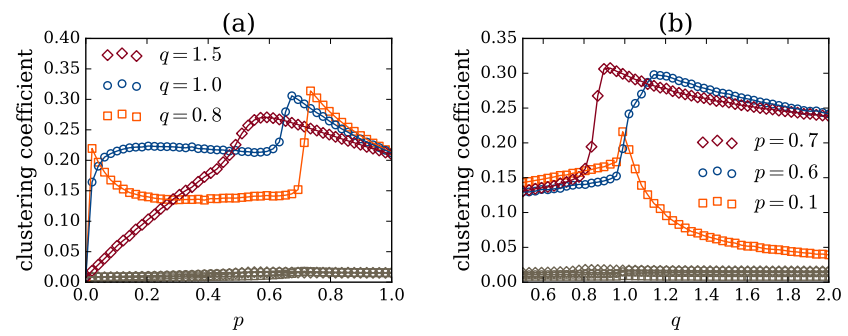

Fig. 6: Average local clustering coefficient $\langle C\rangle$ as a function of (a) $p$ and (b) $q$, on ER networks with $N=1000$, $M=4000$, averaged over 500 simulation runs. Every quantity for global rewiring 35] is also plotted with the same symbols in gray.

Discussion. - In this paper, we have studied a coevolving nonlinear voter model with local rewiring. We identify three different phases, namely consensus, fragmented and dynamically active shattered phases, characterized by different topological structures and their global magnetization. We also examine the transitions between these phases in terms of the size of the largest cluster, the number of clusters, and the magnetization. We find an active shattered phase with $q<1$ and $p<p_{c}$ where the majority of nodes are isolated while a large cluster remains active. This distinct phase which is not detected in global rewiring implies that local rewiring might be the origin of many isolated parts in complex adaptive systems, such as social systems. It would be also interesting [42, 49, 50] to investigate the influence of noise that is a source of randomness, and multilayer structures with the nonlinearity and/or triadic closure [34].

$$
* * *
$$

We acknowledge financial support from the Agencia Estatal de Investigaci on (AEI, Spain) and Fondo Europeo de Desarrollo Regional under project ESOTECOS FIS201563628-C2-2-R (MINECO/AEI/FEDER,UE). This work was supported by the National Research Foundation of Korea (NRF) grant funded by the Korea government (MSIT) (No. 2018R1C1B5044202).

\section{*. - REFERENCES}

[1] Dorogovtsev S. N. Mendes J. F., Advances in physics, 51 (2002) 1079.

[2] Boccaletti S., Latora V., Moreno Y., Chavez M. Hwang D.-U., Physics reports, 424 (2006) 175.

[3] Barrat A., Barthelemy M. Vespignani A., Dynamical processes on complex networks (Cambridge university press) 2008.

[4] Albert R. Barabási A.-L., Reviews of modern physics, $\mathbf{7 4}$ (2002) 47. 
[5] Castellano C., Fortunato S. Loreto V., Reviews of modern physics, 81 (2009) 591.

[6] Zimmermann M. G., Eguiluz V. M. San Miguel M., Cooperation, adaptation and the emergence of leadership in Economics with heterogeneous interacting agents (Springer) 2001 pp. 73-86.

[7] Zimmermann M. G., Eguíluz V. M. San Miguel M., Physical Review E, 69 (2004) 065102.

[8] Vazquez F., Eguíluz V. M. San Miguel M., Physical review letters, 100 (2008) 108702.

[9] Gross T., Dlima C. J. D. Blasius B., Physical review letters, 96 (2006) 208701.

[10] Holme P. Newman M. E., Physical Review E, 74 (2006) 056108.

[11] Gross T. Blasius B., Journal of the Royal Society Interface, 5 (2008) 259.

[12] Biely C., Hanel R. Thurner S., The European Physical Journal B-Condensed Matter and Complex Systems, 67 (2009) 285.

[13] Toruniewska J., Suchecki K. HoŁyst J. A., Physica A: Statistical Mechanics and its Applications, 460 (2016) 1.

[14] Toruniewska J., KuŁakowski K., Suchecki K. HoŁyst J. A., Physical Review E, 96 (2017) 042306.

[15] Marceau V., NoëL P.-A., Hébert-Dufresne L., Allard A. Dubé L. J., Physical Review E, 82 (2010) 036116.

[16] Scarpino S. V., Allard A. HÉbert-Dufresne L., Nature Physics, 12 (2016) 1042.

[17] Vazquez F., Serrano M. Á. San Miguel M., Scientific reports, 6 (2016) 29342.

[18] Vazquez F., González-Avella J. C., Eguíluz V. M. San Miguel M., Phys. Rev. E, 76 (2007) 046120 .

[19] Centola D., Gonzalez-Avella J. C., Eguiluz V. M. San Miguel M., Journal of Conflict Resolution, 51 (2007) 905.

[20] Gracia-Lázaro C., Quijandría F., Hernández L., Floría L. M. Moreno Y., Physical Review E, 84 (2011) 067101.

[21] Raducha T. Gubiec T., Physica A: Statistical Mechanics and its Applications, 471 (2017) 427.

[22] Jordano P., Bascompte J. Olesen J. M., Ecology letters, 6 (2003) 69.
[23] Guimarães Jr P. R., Jordano P. Thompson J. N., Ecology letters, 14 (2011) 877.

[24] Clifford P. Sudbury A., Biometrika, 60 (1973) 581.

[25] Holley R. A. Liggett T. M., The annals of probability, (1975) 643.

[26] Suchecki K., Eguíluz V. M. San Miguel M., Physical Review E, 72 (2005) 036132.

[27] Newman M. E. Park J., Physical Review E, 68 (2003) 036122.

[28] Lee D., Goh K.-I., Kahng B. Kim D., Phys. Rev. E, (2010) 026112.

[29] Klimek P. Thurner S., New Journal of Physics, 15 (2013) 063008.

[30] Bianconi G., Darst R. K., Iacovacci J. Fortunato S., Physical Review E, 90 (2014) 042806.

[31] Holme P. Kim B. J., Physical review E, 65 (2002) 026107.

[32] Davidsen J., Ebel H. Bornholdt S., Physical Review Letters, 88 (2002) 128701.

[33] Klimek P., Diakonova M., Eguíluz V. M., San Miguel M. Thurner S., New Journal of Physics, 18 (2016) 083045.

[34] Diakonova M., San Miguel M. Eguíluz V. M., Physical Review E, 89 (2014) 062818.

[35] Min B. San Miguel M., Scientific Reports, 7 (2017) 12864.

[36] Lambiotte R. Redner S., EPL (Europhysics Letters), 82 (2008) 18007.

[37] Schweitzer F. Behera L., The European Physical Journal B, 67 (2009) 301.

[38] Castellano C., Muñoz M. A. Pastor-Satorras R., Physical Review E, 80 (2009) 041129.

[39] Nyczka P., Sznajd-Weron K. CisŁo J., Physical Review E, 86 (2012) 011105.

[40] Jedrzejewski A., Physical Review E, 95 (2017) 012307.

[41] Radosz W., Mielnik-Pyszczorski A., Brzezińska M. SznAJD-Weron K., Physical Review E, 95 (2017) 062302.

[42] Peralta A., Carro A., Miguel M. S. Toral R., arXiv preprint arXiv:1803.06861, (2018).

[43] Nowak A., Szamrej J. Latané B., Psychological Review, 97 (1990) 362. 
[44] Abrams D. M. Strogatz S. H., Nature, 424 (2003) 900 .

[45] Vazquez F., Castelló X. San Miguel M., Journal of Statistical Mechanics: Theory and Experiment, 2010 (2010) P04007.

[46] Nettle D., Lingua, 108 (1999) 95.

[47] ERdős P. RÉNyi A., Publ. Math. Inst. Hungar. Acad. Sci, 5 (1960) 17.

[48] Watts D. J. Strogatz S. H., nature, 393 (1998) 440.

[49] Diakonova M., Eguíluz V. M. San Miguel M., Physical Review E, 92 (2015) 032803.

[50] Carro A., Toral R. San Miguel M., Scientific reports, 6 (2016) 24775. 